\title{
A systematic review on biomechanical characteristics of walking in children and adolescents with overweight/obesity: possible implications for the development of musculoskeletal disorders
}

Pablo Molina-Garcia ${ }^{1,2 *}$, Jairo H Migueles ${ }^{1}$, Cristina Cadenas-Sanchez ${ }^{1}$, Irene EstebanCornejo ${ }^{1,3}$, Jose Mora-Gonzalez ${ }^{1}$, Maria Rodriguez-Ayllon ${ }^{1}$, Abel Plaza-Florido ${ }^{1}$, Jos Vanrenterghem $^{2}$, Francisco B Ortega ${ }^{1,4}$

${ }^{1}$ PROFITH "PROmoting FITness and Health through physical activity" research group, Department of Physical Education and Sports, Faculty of Sport Sciences, University of Granada, Granada, Spain.

${ }^{2}$ Department of Rehabilitation Sciences, KU Leuven - University of Leuven, Belgium

${ }^{4}$ Center for Cognitive and Brain Health, Department of Psychology, Northeastern University, Boston, MA, USA.

${ }^{4}$ Department of Biosciences and Nutrition, Karolinska Institutet, Sweden

Keywords: Pediatric Obesity, Osteoarthritis, Gait Analysis, Cumulative Trauma Disorders, Energy Metabolism.

Running title: Childhood obesity and biomechanics of walking.

Acknowledgements: We are grateful to Mr. Daniel Gemar García for the illustrations.

Corresponding author: *Pablo Molina-Garcia, Department of Physical and Sports Education, Faculty of Sports Science, University of Granada, Carretera de Alfacar s/n, Granada 18071, Spain. Tel. +34 958 244353. E-mail: pablomolinag5@ugr.es

Potential conflicts of interest: None of the authors have any conflict of interests. 


\section{Summary}

It is known that obesity is associated with biomechanical alterations during locomotor tasks, which is considered a potential risk factor for the development of musculoskeletal disorders (MSKD). However, the association of obesity with biomechanical alterations of walking in the early stages of life have not yet been systematically reviewed. Thus, this review aims to summarise the biomechanical characteristics of walking in children and adolescents with overweight/obesity (OW/OB) versus their normal-weight (NW) counterparts. PubMed and Web of Science were systematically searched until November 2018. We found strong and moderate evidence supporting biomechanical differences in the gait pattern of $\mathrm{OW} / \mathrm{OB}$ with respect to NW. Based on strong evidence, the gait patterns of OW/OB present greater pelvis transversal plane motion, higher hip internal rotation, higher hip flexion, extension and abduction moments and power generation/absorption, greater knee abduction/adduction motion, and higher knee abduction/adduction moments and power generation/absorption. Based on moderate evidence, OW/OB walk with greater step width, longer stance phase, higher tibiofemoral contact forces, higher ankle plantarflexion moments and power generation and greater gastrocnemius and soleus activation/forces. These biomechanical alterations during walking in $\mathrm{OW} / \mathrm{OB}$ could play a major role in the onset and progression of MSKD.

\section{Introduction}

The World Health Organization (WHO) considers obesity in childhood as "one of the most

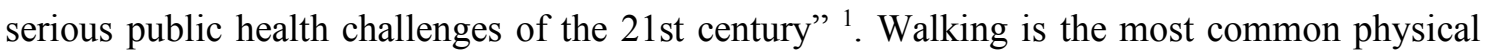
activity in our daily life, and thus, increasing the daily number of steps in children and adolescents has received considerable attention for combating the obesity epidemic ${ }^{2,3}$. Obesity is known to be associated with biomechanical alterations in the gait pattern, which may predispose children and adolescents with overweight or obesity $(\mathrm{OW} / \mathrm{OB})$ to short- and long-term musculoskeletal disorders (MSKD) ${ }^{4-6}$. From early childhood, OW/OB has been associated to the development of various MSKD (i.e., musculoskeletal pain, injuries and fractures) ${ }^{6}$ which may be extended to adulthood with notable consequences with regard to physical disability, quality of life and healthcare economic costs ${ }^{7,8}$. Among other suggested explanations, increased joint loads, together with biomechanical alterations during locomotor tasks, may be underlying the higher prevalence of MSKD in this population ${ }^{4,9,10}$. Furthermore, previous research has revealed that OW/OB show energetic inefficiency during walking, which could be partially explained by a biomechanically inefficient gait pattern ${ }^{9,11,12}$. Altogether, the increased MSKD and an energetic inefficiency 
during walking could be key to the loss of motivation to be physically active, creating a vicious circle which aggravates health issues associated with this population ${ }^{10,13}$.

Recent technological advances in motion capture systems allow accurate assessment of complex biomechanical parameters, which has the potential to provide a comprehensive observation of human movement patterns. These advances have allowed some studies to report numerous gait biomechanical parameters of OW/OB compared to children and adolescents with normal-weight (NW), such as spatiotemporal data (e.g., gait speed or cadence), kinematics (e.g., joint angles or range of motion), kinetics (e.g., joint moments or joint power generation), centre of mass parameters (e.g., velocity, or displacement) or muscle activation and force parameters. In this regard, a previous systematic review studied the biomechanical alterations during walking in adults with obesity ${ }^{14}$, but to the best of our knowledge, the biomechanical alterations in early stages of life have not yet been systematically reviewed. Thus, the aim of this systematic review was to examine the biomechanical characteristics of the gait pattern in OW/OB versus NW.

\section{Methods}

For those readers less familiar with the biomechanical terminology, Table 1 provides definitions of the main biomechanical terms used in this review. Our reviewing procedures were guided by the Preferred Reporting Items for Systematic Reviews and Meta-Analysis (PRISMA) ${ }^{15}$ and the review protocol was registered in the International Prospective Register of Systematic Reviews (https://www.crd.york.ac.uk/prospero/) with reference number: CRD42017067072.

\subsection{Data sources and search strategy}

A systematic literature search was performed in PubMed and Web of Science encompassing publications from inception to November $12^{\text {th }}, 2018$. The search strategy was defined by two reviewers with experience in biomechanics and gait pattern analysis (J-V and P-MG) and two additional reviewers with experience in database searching (C-CS and J-HM). The complete search strategies used for each database are available in Table S1.

\subsection{Eligibility criteria}

Inclusion criteria were defined as follows: 1) participants $\leq 18$ years old; 2) intervention, prospective longitudinal, and cross-sectional articles, written in English, and without any special requirement of sample size; and 3) studies which compared spatiotemporal, kinematics, kinetics, centre of mass or muscle activation/forces parameters of gait between OW/OB and NW. After verifying that there were no intervention and prospective longitudinal studies published on this topic, only cross-sectional articles were included in this systematic review. 
Exclusion criteria were defined as follows: 1) special populations (e.g., participants with movement pattern disorders, musculoskeletal disorders, or pain); 2) meeting abstracts, editorials, letters to editor and reviews; and 3) studies which evaluated gait while carrying extra weight (e.g., participants carrying a backpack). Plantar pressure assessments during gait were beyond the scope of this review and were also not included.

\subsection{Study selection}

The selection process of relevant articles was performed in two stages by two independent researchers (PM-G and AP-F). In the first stage, studies identified in the database search process were introduced to the Web-based systematic review software package "Covidence" (Veritas Health Innovation), which detected duplicate articles. Once the duplicates were deleted from the database, titles and abstracts were examined to identify those likely to be included. In the second stage, full-text of the remaining articles were checked for the final inclusion or exclusion decision. The researchers applied the eligibility criteria at both stages, and disagreements about study selection were resolved in a consensus meeting. Finally, reference lists of included articles were checked for further studies meeting the inclusion criteria, but none were found.

\subsection{Data extraction}

The selection of the data to be extracted was done by one experienced researcher in the field of human biomechanics $(\mathrm{J}-\mathrm{V})$, whereas the subsequent extraction process was done by one researcher (P-MG) and double-checked by two independent researchers (J-HM and C-CS). Defined items to extract were: 1) study reference; 2) biomechanical outcomes measured; 3) sample characteristics; 4) criteria for classification of overweight and obesity; 5) biomechanical instruments used for the assessment; 6) gait assessment protocol characteristics; and 7) main results. When studies included multiple gait analysis conditions (i.e., different treadmill inclinations, walking speeds and illumination), only data from normal conditions were extracted (i.e., no inclination, self-selected walking speed and normal light condition). The joint moments belonging to the kinetic parameters were presented as net internal moments. Disagreement between the reviewers in regards to the extracted data was discussed until consensus was reached.

\subsection{Quality assessment}

The quality assessment of the selected studies was conducted with The Joanna Briggs Institute Critical Appraisal Tool for Systematic Reviews (Table S2) ${ }^{16}$ as used by previous authors ${ }^{17,18}$. This tool was specifically designed to assess quality in cross-sectional studies, and consists of 8 items, each of them with three possible answers as follows: 'yes' (criterion met), 'no' (criterion not met) and 'not applicable'. Whilst potentially ambiguous due to unequal weightings between criteria, a total quality score was calculated for each study to provide a general indication of 
quality. This was done by dividing the number of positively scored (i.e., 'yes') criteria by the total number of applicable criteria. A study was considered as 'high quality' when the quality score was at least 0.75 (i.e., 75\%), whereas studies were considered as 'low quality' when the quality score was lower than $0.75^{18}$. Furthermore, a summary score of each criterion was calculated, by dividing the number of positively scored by the total number of included studies (i.e., 25), to provide an overview of how well the current literature scores on each criterion. Two independent researchers (P-MG and A-PF) accomplished this process, and disagreements were discussed to reach consensus.

\subsection{Evidence synthesis}

Due to the diversity of outcomes from the main biomechanical parameters of gait, a quantitative meta-analysis was not undertaken. We therefore conducted a qualitative evidence synthesis, structuring the evidence in those studies reporting significant differences between OW/OB and NW against those studies reporting no significant differences. The level of evidence was rated similarly to previous literature ${ }^{17,18}$, which considered the number of included studies, their methodological quality and the consistency of findings. Findings were considered consistent if at least $75 \%$ of results pointed in the same direction, showing significant or non-significant differences between $\mathrm{OW} / \mathrm{OB}$ and NW (significance defined as $\mathrm{P}<0.05$ ). The rating protocol was used in previous studies ${ }^{17,18}$, and is described as follows:

Strong evidence: consistent findings in multiple $(\geq 2)$ high-quality studies, pooled with findings from low-quality studies if any existed.

Moderate evidence: consistent findings in 1 high-quality study and at least 1 low-quality study, consistent findings in multiple $(\geq 2)$ low-quality studies or consistent findings in multiple $(\geq 2)$ high-quality studies but inconsistent findings when low-quality studies are considered.

Inconsistent evidence: inconsistent findings in multiple $(\geq 2)$ studies.

Insufficient evidence: only one study available.

\section{Results}

The database search revealed a total of 2,704 articles, of which 353 were duplicates. The titles and abstracts of the remaining 2,351 articles were independently screened by the two researchers, finally including a total of 47 articles which needed a full-text screening. After the full-text screening, 25 cross-sectional articles were deemed to meet the inclusion criteria and were subsequently included in this review. The detailed study selection process is shown in Figure 1.

\subsection{Study and sample characteristics}


Table S3 describes the characteristics of the 25 included studies. Sample sizes of the included studies ranged from 14 to 111 participants. Participants' ages in the included studies ranged from 8 to 18 years old, and the majority of them (73\%) were focused on children (i.e., $\geq 8$ and $\leq 11$ years old) while the remainder (27\%) were focused on adolescents (i.e., $\geq 12$ and $\leq 18$ years old). All included studies were cross-sectional and included different weight categories: underweight (4\%), normal-weight (100\%), overweight (32\%) and obesity (76\%). Regarding the criteria to classify participants as underweight, NW, OW or OB, $48 \%$ of the included studies used the WHO BMI z-scores ${ }^{19}, 28 \%$ used the extended international World Obesity Federation criteria, formerly named as International Obesity Task Force (IOTF) BMI cut-offs ${ }^{20}, 12 \%$ used the Centers for Disease Control and Prevention growth charts for age and sex ${ }^{21}, 4 \%$ used body fat percentiles, and $8 \%$ did not report any criterion.

\subsection{Quality assessment}

Inter-rater reliability for the initial agreement between both researchers (PM-G and AP-F) was high to very high $(\kappa=0.79)$. Among the 25 articles included, $68 \%$ were categorized as 'high quality' and 32\% as 'low quality'. Table $\mathbf{S 2}$ shows the percentage of studies meeting the quality criteria, whereas Table $\mathbf{S} 4$ provides detailed information on the quality score of each study.

\subsection{Biomechanical characteristics of $\mathrm{OW} / \mathrm{OB}$}

A summary of the evidence of gait biomechanics differences between OW/OB and NW is presented in Table 2. Furthermore, a schematic summary of main results based on strong and moderate evidence reporting gait biomechanical differences between OW/OB and NW is presented in Figure 2. These results were classified into the previously mentioned gait biomechanical parameters (i.e., spatiotemporal, joints kinematics and kinetics, centre of mass and muscle activation/force), and also divided into the gait phases and tasks proposed by Perry et al. ${ }^{22}$ and Whittle et al. ${ }^{23}$ (Figure S1). Furthermore, Table S5 provides quantitative information on how large differences presented in Figure 2 were expressed in their original absolute units and as standardized effect sizes (i.e., Cohen's D). Lastly, in order to summarize the information provided in this section, only biomechanical characteristics of gait in $\mathrm{OW} / \mathrm{OB}$ during the main gait phases (i.e., whole gait cycle, stance phase and swing phase) are presented below. To further scrutinize these biomechanical characteristics during all gait phases and tasks, please refer to Figure 2 and Table S5.

\subsubsection{Spatiotemporal parameters}

We found moderate evidence to support that OW/OB walk with greater step width ${ }^{24-27}$ and prolonged stance phase ${ }^{27-31}$ in comparison with NW. On the contrary, there was moderate evidence for non-significant differences between OW/OB and NW in the stride length ${ }^{24,25,28,29,32}$. 
The remaining spatiotemporal parameters (i.e., gait speed, cadence, double support, swing and single support phases) demonstrated an inconsistent level of evidence.

\subsubsection{Upper extremities kinematic and kinetic parameters}

Given that only one study investigated differences between OW/OB and NW in upper extremity kinematics of gait, the level of evidence was set as insufficient. Notwithstanding, this study found that $\mathrm{OW} / \mathrm{OB}$ walk with greater arm motion in the frontal plane, while they display less arm and shoulder motion in the sagittal plane ${ }^{33}$.

\subsubsection{Pelvis and hip kinematic and kinetic parameters}

We found strong evidence that $\mathrm{OW} / \mathrm{OB}$ have greater pelvis transversal plane motion and higher hip internal rotation across the entire gait cycle compared to their NW peers ${ }^{34,35}$. However, we did not find unanimity in kinematic results in the sagittal and frontal planes, and therefore the level of evidence was set as inconsistent. With regard to kinetic parameters, there was strong evidence supporting higher hip flexion, extension and abduction moments and power generation/absorption in $\mathrm{OW} / \mathrm{OB}$ compared to $\mathrm{NW}{ }^{29,35-38}$. Kinetics of transversal plane showed inconsistent evidence, while there was insufficient evidence, with only one study available ${ }^{39}$, to determine differences between $\mathrm{OW} / \mathrm{OB}$ and $\mathrm{NW}$ in terms of contact forces acting on the femoral head during walking.

\subsubsection{Knee kinematic and kinetic parameters}

Analysing the whole gait cycle, we found moderate evidence supporting greater knee frontal plane motion and a knee abducted position for OW/OB in comparison with $\mathrm{NW}{ }^{29,40,41}$. Moreover, kinematic results from the sagittal plane demonstrated inconsistent evidence, and there was insufficient evidence on transverse plane kinematics to determine differences between $\mathrm{OW} / \mathrm{OB}$ and NW. Regarding kinetic parameters, we found moderate evidence supporting higher knee flexion and extension moments and higher power generation/absorption in OW/OB than in NW 29,36-38,42, as well as moderate evidence supporting higher knee abduction and adduction moments and power generation/absorption ${ }^{29,36-38,42,43}$. Furthermore, we found moderate evidence indicating higher tibiofemoral compressive forces and a more medially loaded distribution in OW/OB compared to NW during the stance phase of gait ${ }^{44,45}$. Lastly, there was insufficient evidence on transverse plane knee kinetics with only one study reporting on this ${ }^{37}$.

\subsubsection{Ankle and foot kinematic and kinetic parameters}

Over the whole gait cycle, there was inconsistent evidence reporting differences of ankle and foot kinematics in all three planes (i.e., sagittal, frontal and transverse). Concerning kinetic parameters, we found moderate evidence for higher ankle plantarflexion moments and power generation in OW/OB with respect to NW $28,36-38,43$. Differences between OW/OB and NW in frontal plane 
kinetics were inconsistent, whereas insufficient evidence was available on transverse plane kinetics ${ }^{37}$.

\subsubsection{Centre of mass parameters}

We identified only one study investigating differences in centre of mass parameters between $\mathrm{OW} / \mathrm{OB}$ and NW during walking ${ }^{46}$, indicating an insufficient level of evidence. In this single study, OW/OB showed a lower centre of gravity vertical acceleration during the stance phase in comparison with NW. Moreover, during the stance phase, they displaced their centre of mass with lower velocities than NW in vertical and anterior-posterior directions, whereas during the swing phase they demonstrated a greater centre of mass lateral displacement.

\subsubsection{Muscle activation and forces parameters}

Analysing the whole gait cycle, there was moderate evidence indicating that OW/OB have higher activation and generate higher forces with gastrocnemius and soleus muscles compared to NW 47,48. On the other hand, there was moderate evidence supporting no differences between OW/OB and NW on quadriceps ${ }^{39,47,49}$ and hamstring muscle ${ }^{39,49}$ activations and forces, respectively. The remaining muscles studied in the gait pattern of $\mathrm{OW} / \mathrm{OB}$ and NW were inconsistent (i.e., gluteus complex) or insufficient (i.e., psoas, iliacus and tibialis anterior).

\section{Discussion}

In the present systematic review, we provide an overview of the biomechanical characteristics of gait in $\mathrm{OW} / \mathrm{OB}$ with respect to $\mathrm{NW}$, based on a systematic review of the literature. The main findings of this study were: 1) based on strong evidence, the gait patterns of OW/OB present greater pelvis transversal plane motion, higher hip internal rotation, higher hip flexion, extension and abduction moments and power generation/absorption, greater knee abduction/adduction motion, and higher knee abduction/adduction moments and power generation/absorption; and 2) based on moderate evidence, $\mathrm{OW} / \mathrm{OB}$ walk with greater step width, longer stance phase, higher tibiofemoral contact forces, higher ankle plantarflexion moments and power generation and greater gastrocnemius and soleus activation/forces.

\subsection{Spatiotemporal parameters}

Among all spatiotemporal parameters of gait studied, we only found consistent results between studies supporting that OW/OB walk with a greater step width and longer stance phase than their NW peers. These characteristics could arise from a necessity to generate added stability by walking with a greater base of support in the frontal plane, expressed through a wider step ${ }^{50}$. Lengthening the stance phase could also indicate a poorer overall stability in this population, likely accompanied by a greater difficulty decelerating and reaccelerating their body mass for the 
next step ${ }^{28,51,52}$. Concerning the remaining spatiotemporal parameters, there were no consistent differences between OW/OB and NW. Further studies should determine whether the presence of overweight/obesity has an effect on these parameters, preferably under non-laboratory conditions (e.g., pedometry or accelerometry) where children tend to present a more natural gait pattern.

\subsection{Pelvis and hip kinematics and kinetics}

Pelvis and hip kinematics of OW/OB only showed differences with respect to NW in the transverse plane, with increased pelvis transverse plane motion and hip internal rotation. This increased pelvis motion has been associated with a lack of stability and motor control in the lumbopelvic region ${ }^{53}$. The increased hip internal rotation in this population could be due to the adipose tissue accumulated between their thighs hampering movement in the sagittal plane and requiring compensations in the transverse plane ${ }^{27,54}$. With regard to kinetics, OW/OB generated higher hip extension and abduction power, possibly to prevent lower-limb collapse and maintain an upright posture ${ }^{28,38}$. Some authors demonstrated that these altered power generation patterns remained after accounting for body mass, which could indicate a locomotor adaptation to walking with extra weight ${ }^{36}$.

On the other hand, results from the pelvis and hip kinematics in the sagittal and frontal plane, as well as kinetics in the transverse plane, were inconsistent. To date, one of the main limitations of gait analysis in OW/OB is the presence of soft tissue artefacts in the data, especially around the pelvis and thigh where this population accumulates greater adiposity ${ }^{55}$. Only studies of Briggs et al. ${ }^{42}$, Lerner et al. ${ }^{39,45}$ and Strutzenberger et al. ${ }^{29}$ addressed soft tissue artefacts, by using virtual markers relative to anatomical structures less likely to present adiposity (e.g., the sacrum), or by measuring the distance between anatomical landmarks (e.g., left and right anterior superior iliac spines) in order to recreate the adequate position of markers. Possibly this limitation, together with the use of different methodologies to measure kinematic and kinetic parameters, can partially explain the inconsistencies observed at the pelvis and hip. We could only identify one study which investigated the influence of paediatric obesity on hip joint contact forces during walking ${ }^{39}$. Lerner et al. ${ }^{39}$ found that $\mathrm{OW} / \mathrm{OB}$ are exposed to higher femoral head forces in compressive and anterior-posterior directions compared to NW during the gait pattern. Further research is warranted to confirm these higher hip contact forces in $\mathrm{OW} / \mathrm{OB}$, and to propose gait analysis procedures to minimize soft tissue artefacts in this specific population.

\subsection{Knee kinematics and kinetics parameters}

An important finding of this review is the presence of knee kinematic and kinetic alterations observed in OW/OB during walking, which consist of greater knee frontal plane motion and higher knee frontal plane moments and power generation/absorption. These results might be linked to the greater step width and hip internal rotation position above mentioned, which together 
could be indicating a lower limb valgus position commonly adopted by OW/OB during gait ${ }^{37}$. Some authors have suggested that this valgus position helps provide a better dynamic stability in the frontal plane during gait, and that it could be a subconscious strategy to reduce loading on the medial compartment of the knee joint ${ }^{29,34}$. In agreement with the aforementioned findings in pelvis and hip, OW/OB still presented greater knee abduction power absorption than NW after taking into account their body mass, suggesting again that gait biomechanical alterations of this population are not only explained by the presence of excessive body mass ${ }^{29,36}$. We did not find consistent evidence of knee kinematic and kinetic differences in the sagittal plane of OW/OB with respect to NW, whereas there was insufficient literature in the transverse plane. The available literature suggests that $\mathrm{OW} / \mathrm{OB}$ present a knee externally rotated position and higher knee transverse plane moments and power generation/absorption, nonetheless future studies will need to corroborate these findings ${ }^{34,37}$.

Concerning tibiofemoral contact forces, OW/OB have demonstrated higher absolute compressive forces and, furthermore, they seem to walk with a more medial tibiofemoral load distribution ${ }^{45,48}$. To further explore how obesity affects knee contact forces relative to the skeletal structure, Lerner et al. ${ }^{45}$ accounted for physis cross-sectional area in their analysis, and they discovered that the medial tibiofemoral forces were still 1.77 times greater in OW/OB. It could imply that OW/OB not only have higher medial tibiofemoral forces than their NW peers, but also that their skeletal structure is not adapted to supporting the greater mechanical stresses ${ }^{45}$.

\subsection{Ankle and foot kinematics and kinetics parameters}

Concerning ankle and foot biomechanics during the whole gait cycle, we only found consistent evidence supporting higher ankle plantarflexion moments and power generation in OW/OB compared to NW. Some authors have acknowledged this compensation as a need to decelerate and propel their heavier body mass into the next step ${ }^{28,38}$. Ankle and foot kinematics in all three planes, as well as kinetics in the frontal plane, demonstrated inconsistent results. In this sense, it is important to highlight that only Mahaffey et al. ${ }^{56}$ took into account the complex motion of the multiple foot segments in their study, whereas the rest of the authors considered the foot as a single rigid segment. Future studies should specifically focus on ankle and foot biomechanics during gait in this population from a multiple segments perspective, with a special emphasis on kinematic parameters.

\subsection{Muscle activation and forces}

This systematic review evidences a higher activation and forces of the gastrocnemius and soleus complex in OW/OB than NW during the whole gait cycle, which is in agreement with the higher ankle plantarflexion moment and power generation previously mentioned ${ }^{45}$. Furthermore, some authors revealed that higher body fat percentage was associated with higher soleus forces 
independently of participants' muscle mass, which reveals a real hyper-activation of this muscle during walking since this population has demonstrated not only greater body mass, but also greater muscle mass ${ }^{47,57}$. Additionally, the fact that, after adjusting for body mass, OW/OB displayed lower forces in gastrocnemius and soleus muscle complexes, denotes the importance of excess of weight in the greater muscle force requirements ${ }^{47,48}$.

On the other hand, quadriceps and hamstring muscles have demonstrated a similar activation and forces profile in both $\mathrm{OW} / \mathrm{OB}$ and $\mathrm{NW}$, results which seem to not be consistent with the abovementioned higher hip and knee kinetic values in OW/OB. Among the remaining muscles studied, the gluteus complex showed inconsistent results whereas psoas iliacus and tibialis anterior presented insufficient evidence to draw solid conclusions. On the basis of the above, further investigation is necessary to determine whether muscle activation patterns during walking between $\mathrm{OW} / \mathrm{OB}$ and $\mathrm{NW}$ are meaningfully different.

\subsection{Clinical implications}

\subsubsection{Development of musculoskeletal disorders}

Findings from this systematic review support the belief that gait biomechanical alterations observed in OW/OB could have harmful implications on their musculoskeletal system. This population has demonstrated a higher prevalence of MSKD, especially in lumbar, hip and knee regions $5,6,13$. Interestingly, our systematic review revealed that $\mathrm{OW} / \mathrm{OB}$ present kinematic and kinetic alterations in these regions while walking, which could be a factor to consider in the onset and development of MSKD in this population. Lower limb kinematic alterations during locomotor tasks, such as those found in this review, are considered a risk factor for the development of osteoarthritis through a progressive degeneration of articular cartilage and soft tissues ${ }^{4}$. Moreover, higher contact forces applied on the hip and knee joints could partially explain the increased prevalence of hip and knee pain in this population, as well as the long-term development of osteoarthritis ${ }^{39,58}$.

It is important to note that these gait biomechanical alterations have been reported during walking, where individuals must typically endure peak loads of 1.2 times their body weight, whereas in other activities such as running or jumping, the loads can increase 2 to 5 times body weight ${ }^{59,60}$. If the biomechanical alterations observed in walking would also extend to higher intensity physical activities, OW/OB could experience an even greater risk for developing MSKD. The gait patterns of $\mathrm{OW} / \mathrm{OB}$ suggested in this review present similarities with other populations suffering from MSKD. For instance, some authors have reported that patients undergoing low back pain present increased pelvis transverse plane motion during walking ${ }^{61}$, whereas patients present with knee osteoarthritis also walk with higher knee adduction moments than healthy-patients ${ }^{62}$. 
Among other possible consequences, the development of MSKD will likely decrease motivation of OW/OB to be physically active, leading them into a downwards spiral of accelerated disease progression ${ }^{10,13}$. This creates a huge direct burden on society ${ }^{63}$, but has also major implications for mobility and quality of life during adulthood and old age ${ }^{8,64}$. It is important to highlight that all associations between biomechanical alterations and MSKD proposed in this review are based on hypotheses. Longitudinal investigation is needed to determine whether gait biomechanical alterations in OW/OB predict the future development of MSKD.

\subsubsection{Energetic inefficiency of walking}

The energetic cost of walking, described as the metabolic rate required to walking at a given speed, is greater in $\mathrm{OW} / \mathrm{OB}$ compared to $\mathrm{NW}^{11,12}$. Obviously, the need to carry extra weight while walking is a primary determinant of the elevated energy expenditure in this population, however, a biomechanically inefficient gait pattern has been proposed as an additional factor ${ }^{11,65}$. For instance, a greater step width, as has been evidenced in $\mathrm{OW} / \mathrm{OB}$, is related to an increased energetic cost in human walking ${ }^{11,66}$. In addition, higher joint moments and powers, as well as an increased demand for muscle activation, suggest higher energy expenditure during walking at normal speed ${ }^{27,67}$.

Overall, gait biomechanical alterations shown in this review provide further insight into the roles of greater energetic cost of walking in $\mathrm{OW} / \mathrm{OB}$. Although an elevated walking energy cost may seem beneficial since obesity is an energy imbalance between calories consumed and expended ${ }^{68}$, it also comes with a greater burden on the musculoskeletal system, and a relatively greater effort of walking ${ }^{9}$. The latter could be key in the lack of motivation to be physically active, creating a vicious circle which will aggravate health issues associated with this population.

\subsection{Strengths and limitations}

A strength of this work is the combination of a systematic review of the literature with an evidence synthesis based on methodological quality, which allows us to not only draw conclusions from the included articles but also to establish the level of evidence of our findings. Moreover, we provide two different formats to report our findings: a graphical and schematic summarized figure containing the gait biomechanical characteristics of OW/OB found in this review (Figure 2), and a detailed table providing quantitative information of these characteristics (Table S5).

One limitation of this review is that it only includes cross-sectional studies, due to the lack of intervention and longitudinal studies, preventing any causality conclusions between the presence of excessive body mass and biomechanical alterations during walking. With regard to the search strategy, the use of only two databases (PubMed and Web of Science) may have included out some articles related to this topic. Nevertheless, it is expected that those databases cover any 
influential peer-reviewed journals in which one expects to find relevant articles for this review. We need to acknowledge that there is no current evidence supporting that the biomechanical alterations experienced by OW/OB predict MSKD in adulthood and, therefore, any conclusions around that are based on assumptions and hypotheses. It is also important to acknowledge that the gait pattern is under a development process during childhood, and future research should therefore focus on determining the age at which these biomechanical alterations become apparent in OW/OB. Lastly, the included articles presented considerably different instruments, assessment protocols and data processing methodologies to analyse the gait pattern, a fact which should be acknowledged in future studies in order to make the results more comparable.

\section{Conclusion}

Findings from this review reveal strong and moderate evidence supporting biomechanical differences in the gait pattern of OW/OB with respect to NW. Overall, there was strong evidence that gait patterns of $\mathrm{OW} / \mathrm{OB}$ present greater pelvis transversal plane motion, higher hip internal rotation, higher hip flexion, extension and abduction moments and power generation/absorption, greater knee abduction/adduction motion, and higher knee abduction/adduction moments and power generation/absorption. Furthermore, based on moderate evidence, OW/OB walk with greater step width, longer stance phase, higher tibiofemoral contact forces, higher ankle plantarflexion moments and power generation and greater gastrocnemius and soleus activation/forces. These alterations observed in OW/OB could be determinant in the short and long-term development of MSKD and could be a key factor to understanding the energetic inefficiency experienced by this population during walking.

\section{Supporting information}

Table S1. Search terms used in PubMed and Web of Science databases.

Table S2. Criteria for the methodological quality assessment of included articles and percentage of studies meeting these criteria.

Table S3. Summary of study characteristics of articles included in review.

Table S4. Quality assessment of included articles.

Table S5. Summary of the main gait biomechanical differences between children and adolescents with overweight/obesity and normal-weight divided by key gait phases.

\section{References}

1. Childhood overweight and obesity. World Health Organization (WHO) website https://www.who.int/dietphysicalactivity/childhood/en/. Updated 2017. Accessed 
December 17, 2018.

2. Saavedra JM, Escalante Y, Garcia-Hermoso A. Improvement of aerobic fitness in obese children: a meta-analysis. Int J Pediatr Obes 2011; 6(3-4):169-177.

3. Metcalf B, Henley W, Wilkin T. Effectiveness of intervention on physical activity of children: systematic review and meta-analysis of controlled trials with objectively measured outcomes (EarlyBird 54). BMJ 2012; 345(sep27 1):e5888-e5888.

4. Wearing SC, Hennig EM, Byrne NM, Steele JR, Hills AP. Musculoskeletal disorders associated with obesity: a biomechanical perspective. Obes Rev 2006; 7(3):239-250.

5. Smith SM, Sumar B, Dixon KA. Musculoskeletal pain in overweight and obese children. Int J Obes 2014; 38(1):11-15.

6. Paulis WD, Silva S, Koes BW, van Middelkoop M. Overweight and obesity are associated with musculoskeletal complaints as early as childhood: a systematic review. Obes Rev 2014; 15(1):52-67.

7. Henschke N, Kamper SJ, Maher CG. The Epidemiology and Economic Consequences of Pain. Mayo Clin Proc 2015; 90(1):139-147.

8. Vincent HK, Vincent KR, Lamb KM. Obesity and mobility disability in the older adult. Obes Rev 2010; 11(8):568-579.

9. Shultz SP, Browning RC, Schutz Y, Maffeis C, Hills AP. Childhood obesity and walking: guidelines and challenges. Int J Pediatr Obes 2011; 6(5-6):332-341.

10. Shultz SP, Anner J, Hills AP. Paediatric obesity, physical activity and the musculoskeletal system. Obes Rev 2009; 10(5):576-582.

11. Peyrot N, Thivel D, Isacco L, Morin J-B, Duche P, Belli A. Do mechanical gait parameters explain the higher metabolic cost of walking in obese adolescents? $\mathrm{J} \mathrm{Appl}$ Physiol 2009; 106(6):1763-1770.

12. Ayub BV, Bar-Or O. Energy cost of walking in boys who differ in adiposity but are matched for body mass. Med Sci Sports Exerc 2003; 35(4):669-674.

13. Stovitz SD, Pardee PE, Vazquez G, Duval S, Schwimmer JB. Musculoskeletal pain in obese children and adolescents. Acta Paediatr 2008; 97(4):489-493.

14. Runhaar J, Koes BW, Clockaerts S, Bierma-Zeinstra SM. A systematic review on changed biomechanics of lower extremities in obese individuals: a possible role in development of osteoarthritis. Obes Rev 2011; 12(12):1071-1082.

15. Moher D, Liberati A, Tetzlaff J, Altman DG, PRISMA Group. Preferred Reporting Items for Systematic Reviews and Meta-Analyses: The PRISMA Statement. PLoS Med 2009; 6(7):e1000097.

16. Moola S, Munn Z, Tufanaru C, Aromataris E, Sears K, Sfetcu R, Currie M, Qureshi R, Mattis P, Lisy K MP-F. Chapter 7: Systematic reviews of etiology and risk - Joanna Briggs Institute Reviewers' Manual. Joanna Briggs Inst Rev Man 2017.

17. Haynes A, Kersbergen I, Sutin A, Daly M, Robinson E. A systematic review of the relationship between weight status perceptions and weight loss attempts, strategies, behaviours and outcomes. Obes Rev 2018; 19(3):347-363.

18. van Ekris E, Altenburg TM, Singh AS, Proper KI, Heymans MW, Chinapaw MJ. An evidence-update on the prospective relationship between childhood sedentary behaviour and biomedical health indicators: a systematic review and meta-analysis. Obes Rev 2016; 17(9):833-849.

19. BMI-for-age (5-19 years). World Health Organization (WHO) website https://www.who.int/growthref/who2007_bmi_for_age/en/. Updated 2015. Accessed 
December 17, 2018.

20. Cole TJ, Lobstein T. Extended international (IOTF) body mass index cut-offs for thinness, overweight and obesity. Pediatr Obes 2012; 7(4):284-294.

21. Kuczmarski RJ, Ogden CL, Grummer-Strawn LM, et al. CDC growth charts: United States. Adv Data 2000;(314):1-27.

22. Perry J, Burnfield JM, Cabico LM. Gait Analysis : Normal and Pathological Function. SLACK; 2010.

23. Whittle M. Gait Analysis : An Introduction. Butterworth-Heinemann; 2007.

24. D'Hondt E, Segers V, Deforche B, et al. The role of vision in obese and normal-weight children's gait control. Gait Posture 2011; 33(2):179-184.

25. Deforche BI, Hills AP, Worringham CJ, et al. Balance and postural skills in normalweight and overweight prepubertal boys. Int J Pediatr Obes 2009; 4(3):175-182.

26. Dufek JS, Currie RL, Gouws PL, et al. Effects of overweight and obesity on walking characteristics in adolescents. Hum Mov Sci 2012; 31(4):897-906.

27. Huang L, Chen P, Zhuang J, Zhang Y, Walt S. Metabolic Cost, Mechanical Work, and Efficiency During Normal Walking in Obese and Normal-Weight Children. Res Q Exerc Sport 2014; 84(sup2):S72-S79.

28. Cimolin V, Galli M, Vismara L, Albertini G, Sartorio A, Capodaglio P. Gait pattern in lean and obese adolescents. Int J Rehabil Res 2015; 38(1):40-48.

29. Strutzenberger G, Alexander N, Bamboschek D, Claas E, Langhof H, Schwameder H. Uphill walking: Biomechanical demand on the lower extremities of obese adolescents. Gait Posture 2017; 54:20-26.

30. Hills AP, Parker AW. Locomotor characteristics of obese children. Child Care Health Dev 1992; 18(1):29-34.

31. McGraw B, McClenaghan BA, Williams HG, Dickerson J, Ward DS. Gait and postural stability in obese and nonobese prepubertal boys. Arch Phys Med Rehabil 2000; 81(4):484-489.

32. Nantel J, Brochu M, Prince F. Locomotor Strategies in Obese and Non-obese Children*. Obesity 2006; 14(10):1789-1794.

33. Hung Y-C, Gill S V., Meredith GS. Influence of Dual-Task Constraints on Whole-Body Organization During Walking in Children Who Are Overweight and Obese. Am J Phys Med Rehabil 2013; 92(6):461-471.

34. Shultz SP, D'Hondt E, Fink PW, Lenoir M, Hills AP. The effects of pediatric obesity on dynamic joint malalignment during gait. Clin Biomech 2014; 29(7):835-838.

35. Cimolin V, Galli M, Vismara L, Albertini G, Sartorio A, Capodaglio P. Gait pattern in lean and obese adolescents. Int $J$ Rehabil Res 2015; 38(1):40-48.

36. Shultz SP, Hills AP, Sitler MR, Hillstrom HJ. Body size and walking cadence affect lower extremity joint power in children's gait. Gait Posture 2010; 32(2):248-252.

37. Shultz SP, Sitler MR, Tierney RT, Hillstrom HJ, Song J. Effects of Pediatric Obesity on Joint Kinematics and Kinetics During 2 Walking Cadences. Arch Phys Med Rehabil 2009; 90(12):2146-2154.

38. Shultz SP, D'Hondt E, Lenoir M, Fink PW, Hills AP. The role of excess mass in the adaptation of children's gait. Hum Mov Sci 2014; 36:12-19.

39. Lerner ZF, Browning RC. Compressive and shear hip joint contact forces are affected by pediatric obesity during walking. J Biomech 2016; 49(9):1547-1553. 
40. McMillan AG, Auman NL, Collier DN, Blaise Williams DS. Frontal Plane Lower Extremity Biomechanics During Walking in Boys Who Are Overweight Versus Healthy Weight. Pediatr Phys Ther 2009; 21(2):187-193.

41. McMillan AG, Pulver AME, Collier DN, Williams DSB. Sagittal and frontal plane joint mechanics throughout the stance phase of walking in adolescents who are obese. Gait Posture 2010; 32(2):263-268.

42. Briggs MS, Bout-Tabaku S, McNally MP, Chaudhari AMW, Best TM, Schmitt LC. Relationships between standing frontal-plane knee alignment and dynamic knee joint loading during walking and jogging in youth who are obese. Phys Ther 2017; 97(5):571580 .

43. Gushue DL, Houck J, Lerner AL. Effects of childhood obesity on three-dimensional knee joint biomechanics during walking. J Pediatr Orthop 2005; 25(6):763-768.

44. Huang L, Zhuang J, Zhang Y. The Application of Computer Musculoskeletal Modeling and Simulation to Investigate Compressive Tibiofemoral Force and Muscle Functions in Obese Children. Comput Math Methods Med 2013; 2013:1-10.

45. Lerner ZF, Board WJ, Browning RC. Pediatric obesity and walking duration increase medial tibiofemoral compartment contact forces. J Orthop Res 2016; 34(1):97-105.

46. Colné P, Frelut ML, Pérès G, Thoumie P. Postural control in obese adolescents assessed by limits of stability and gait initiation. Gait Posture 2008; 28(1):164-169.

47. Lerner ZF, Shultz SP, Board WJ, Kung S, Browning RC. Does adiposity affect muscle function during walking in children? J Biomech 2014; 47(12):2975-2982.

48. Huang L, Zhuang J, Zhang Y. The application of computer musculoskeletal modeling and simulation to investigate compressive tibiofemoral force and muscle functions in obese children. Comput Math Methods Med 2013; 2013.

49. Blakemore VJ, Fink PW, Lark SD, Shultz SP. Mass affects lower extremity muscle activity patterns in children's gait. Gait Posture 2013; 38(4):609-613.

50. Browning RC. Locomotion Mechanics in Obese Adults and Children. Curr Obes Rep 2012; 1(3):152-159.

51. Granacher U, Gollhofer A, Kriemler S. Effects of balance training on postural sway, leg extensor strength, and jumping height in adolescents. Res Q Exerc Sport 2010; 81(3):245-251.

52. Tomlinson DJ, Erskine RM, Morse CI, Winwood K, Onambele-Pearson G. The impact of obesity on skeletal muscle strength and structure through adolescence to old age. Biogerontology 2016; 17(3):467-483.

53. Wu WH, Lin XC, Meijer OG, et al. Effects of experimentally increased trunk stiffness on thorax and pelvis rotations during walking. Hum Mov Sci 2014; 33(1):194-202.

54. Deforche B, Hills AP, Worringham CJ, et al. Balance and postural skills in normalweight and overweight prepubertal boys. Int J Pediatr Obes 2009; 4(3):175-182.

55. Horsak B, Schwab C, Clemens C, et al. Is the reliability of 3D kinematics of young obese participants dependent on the hip joint center localization method used? Gait Posture 2018; 59(May 2017):65-70.

56. Mahaffey R, Morrison SC, Bassett P, Drechsler WI, Cramp MC. The impact of body fat on three dimensional motion of the paediatric foot during walking. Gait Posture 2016; 44:155-160.

57. Forbes GB, Welle SL. Lean body mass in obesity. Int J Obes 1983; 7(2):99-107.

58. Sharma L, Song J, Felson DT, Cahue S, Shamiyeh E, Dunlop DD. The role of knee 
alignment in disease progression and functional decline in knee osteoarthritis. JAMA 2001; 286(2):188-195.

59. Cavanagh PR, Lafortune MA. Ground reaction forces in distance running. $J$ Biomech 1980; 13(5):397-406.

60. Jacob HA. Forces acting in the forefoot during normal gait--an estimate. Clin Biomech (Bristol, Avon) 2001; 16(9):783-792.

61. Peng Y, Sjoerd H, Jian MB, Lin H, Meijer OG. Gait adaptations in low back pain patients with lumbar disc herniation : trunk coordination and arm swing. 2011:491-499.

62. Baliunas AJ, Hurwitz DE, Ryals AB, et al. Increased knee joint loads during walking are present in subjects with knee osteoarthritis. Osteoarthr Cartil 2002; 10(7):573-579.

63. Lee IM, Shiroma EJ, Lobelo F, Puska P, Blair SN, Katzmarzyk PT. Effect of physical inactivity on major non-communicable diseases worldwide: an analysis of burden of disease and life expectancy. Lancet 2012; 380(9838):219-229.

64. Musculoskeletal pain. International Association for the Study of Pain (IASP) website https://www.iasp-pain.org/SIG/MusculoskeletalPain?navItemNumber=5268. Updated 2018. Accessed December 17, 2018.

65. Schwartz MH, Koop SE, Bourke JL, Baker R. A nondimensional normalization scheme for oxygen utilization data. Gait Posture 2006; 24(1):14-22.

66. Donelan JM, Kram R, Kuo AD. Mechanical and metabolic determinants of the preferred step width in human walking. Proc Biol Sci 2001; 268(1480):1985-1992.

67. Grabowski A, Farley CT, Kram R. Independent metabolic costs of supporting body weight and accelerating body mass during walking. J Appl Physiol 2005; 98(2):579-583.

68. Bray GA. Medical consequences of obesity. J Clin Endocrinol Metab 2004; 89(6):25832589.

69. Nantel J, Brochu M, Prince F. Locomotor Strategies in Obese and Non-obese Children. Obesity 2006; 14(10):1789-1794.

70. Gushue DL, Houck J, Lerner AL. Effects of Childhood Obesity on Three-Dimensional Knee Joint Biomechanics During Walking. J Pediatr Orthop 2005; 25(6):763-768. 


\section{Tables and figures}

9. Table 1. Definition of the biomechanical parameters of gait used in this review

\begin{tabular}{|c|c|}
\hline Variable & Definition \\
\hline Spatiotemporal & $\begin{array}{l}\text { The study of spatial (distance) and temporal (time) parameters during } \\
\text { gait }\end{array}$ \\
\hline Gait Speed & Walking speed. Is reported in $\mathrm{m} . \mathrm{s}^{-1}$. \\
\hline Stride length & $\begin{array}{l}\text { The interval between the first and second contact of the same foot. Is } \\
\text { reported in } \mathrm{m} \text { or } \mathrm{cm} \text {. }\end{array}$ \\
\hline Step length & The interval between initial contact of each foot. Is reported in $\mathrm{m}$ or $\mathrm{cm}$. \\
\hline Step width & The distance between the lateral margins of the feet. Is reported in $\mathrm{m}$ or $\mathrm{cm}$. \\
\hline Stance phase & $\begin{array}{l}\text { The entire period during which the foot is on the ground. Is reported in a } \\
\text { percentage of gait cycle or in s }\end{array}$ \\
\hline Swing phase & $\begin{array}{l}\text { The entire period during which the foot is in the air. Is reported in a } \\
\text { percentage of gait cycle or in } \mathrm{s}\end{array}$ \\
\hline Single support & $\begin{array}{l}\text { The period in which only one foot is on the floor. It starts when the } \\
\text { opposite foot is lifted and ends with the opposite foot contacting again. Is } \\
\text { reported in a percentage of gait cycle or in s }\end{array}$ \\
\hline Double support & $\begin{array}{l}\text { The period in which both feet are on the floor, starts with the initial contact } \\
\text { of one foot and ends with the lifting of the opposite foot. Is reported in a } \\
\text { percentage of gait cycle or in s. }\end{array}$ \\
\hline Cadence & The number of steps per minute. Is reported in steps per minute \\
\hline
\end{tabular}

\section{Kinematics \\ Joint angular motion \\ Maximum angular motion}

\section{Kinetics \\ Joint moment \\ Joint power \\ generation \\ Joint power \\ absorption \\ Joint compressive force \\ Joint shear force \\ Joint loading rate}

\section{Centre of mass}

Centre of mass velocity and acceleration Centre of mass displacement

\section{Muscle activity/force} Muscle activation

Muscle force
The study of displacement parameters of body segments during gait in the three anatomical planes of motion (sagittal, frontal and transversal) The angular displacement of a joint in a specific anatomical plane of motion (sagittal, frontal and transversal). Is reported in degrees The furthest observed angular displacement of a joint in an anatomical plane of motion (sagittal, frontal and transversal). Is reported in degrees

The study of force parameters associated with body segment motion during gait in the three anatomical planes of motion (sagittal, frontal and transversal)

The net joint rotational effort produced by all muscles spanning a joint. Is reported in N.m.

The rate at which joint work is performed. Has a positive value with the generation of energy, typically associated with concentric muscle activity. Is reported in Watts or Joules per second

The rate at which joint work is performed. Has a negative value with the absorption of energy, typically associated with eccentric muscle activity. Is reported in Watts or Joules per second

Vector force acting perpendicular to the joint surface along the bone's longitudinal axis, which compresses the joint structures. Is reported in Newtons

Vector force acting in parallel with the joint surface, which causing shear stress to the joint structures. Is reported in Newtons

The rate at which joint force increases, typically reported for joint compressive forces. Is reported in Newtons per second

\section{The study of the point representing the mean position of body mass} during gait

The velocity or acceleration of the centre of mass during gait. Is reported in $\mathrm{m} . \mathrm{s}^{-1}$ and $\mathrm{m} . \mathrm{s}^{-2}$, respectively.

The displacement of the centre of mass during gait. Is reported in $\mathrm{m}$ or $\mathrm{cm}$

The study of muscle activity patterns and muscle forces during gait Defined as having an amplitude greater than the mean amplitude plus two standard deviations of a static trial. Is reported as a percentage of gait phases Estimated muscle force from model simulations. Reported in Newtons 
Table 2. Evidence synthesis of gait biomechanical differences between overweight/obese and normal-weight children and adolescents, including article references

\begin{tabular}{|c|c|c|c|c|c|c|c|}
\hline \multirow{2}{*}{$\begin{array}{c}\text { Gait biomechanical } \\
\text { parameters }\end{array}$} & \multirow{2}{*}{ N studies } & \multicolumn{2}{|c|}{$\begin{array}{c}\begin{array}{c}\text { Significant } \\
\text { difference }\end{array} \\
\end{array}$} & \multicolumn{2}{|c|}{$\begin{array}{c}\text { No significant } \\
\text { difference }\end{array}$} & \multirow{2}{*}{$\begin{array}{c}\text { Consistency } \\
\%\end{array}$} & \multirow{2}{*}{$\begin{array}{l}\text { Level of } \\
\text { Evidence }\end{array}$} \\
\hline & & $\begin{array}{l}\text { High } \\
\text { Quality }\end{array}$ & $\begin{array}{c}\text { Low } \\
\text { Quality }\end{array}$ & $\begin{array}{c}\text { High } \\
\text { Quality }\end{array}$ & $\begin{array}{c}\text { Low } \\
\text { Quality }\end{array}$ & & \\
\hline \multicolumn{8}{|l|}{ Spatio-temporals } \\
\hline Gait Speed & $\begin{array}{c}9 \\
24-28,30,32,33,44\end{array}$ & $\begin{array}{c}2 \\
26,27\end{array}$ & $\begin{array}{c}3 \\
30,33,44\end{array}$ & $\begin{array}{c}3 \\
24,25,28\end{array}$ & $\begin{array}{c}1 \\
32\end{array}$ & 56 & Inconsistent \\
\hline Cadence & $\begin{array}{c}6 \\
24,27,29-32\end{array}$ & $\begin{array}{c}1 \\
27\end{array}$ & $\begin{array}{c}1 \\
30\end{array}$ & $\underset{24,29}{2}$ & $\underset{31,32}{2}$ & 67 & Inconsistent \\
\hline Stride length & $\begin{array}{c}8 \\
24,25,27-30,32,33\end{array}$ & $\begin{array}{c}1 \\
27\end{array}$ & $\underset{30,33}{2}$ & $\begin{array}{c}4 \\
24,25,28,29\end{array}$ & $\begin{array}{c}1 \\
32\end{array}$ & 63 & $\begin{array}{l}\text { Moderate } \\
\text { no diff. }\end{array}$ \\
\hline Step width & $\begin{array}{c}6 \\
24-27,29,33\end{array}$ & $\begin{array}{c}4 \\
24-27\end{array}$ & - & $\begin{array}{c}1 \\
29\end{array}$ & $\begin{array}{c}1 \\
33\end{array}$ & 67 & $\begin{array}{l}\text { Moderate } \\
\text { diff. }\end{array}$ \\
\hline Stance phase & $\begin{array}{c}8 \\
24,27-33\end{array}$ & $\begin{array}{c}3 \\
27-29\end{array}$ & $\begin{array}{c}2 \\
30,31\end{array}$ & $\begin{array}{c}1 \\
24\end{array}$ & $\underset{32,33}{2}$ & 62 & $\begin{array}{l}\text { Moderate } \\
\text { diff. }\end{array}$ \\
\hline Double support phase & $\begin{array}{c}6 \\
24,26,27,31,32,46\end{array}$ & $\underset{26,27}{2}$ & $\underset{31,46}{2}$ & $\begin{array}{c}1 \\
24\end{array}$ & $\begin{array}{c}1 \\
32\end{array}$ & 67 & Inconsistent \\
\hline Swing phase & $\begin{array}{c}6 \\
24,26,29-31,46\end{array}$ & $\underset{26,29}{2}$ & $\begin{array}{l}1 \\
31\end{array}$ & $\begin{array}{l}1 \\
24\end{array}$ & $\begin{array}{c}2 \\
30,46\end{array}$ & 50 & Inconsistent \\
\hline Single support phase & $\begin{array}{c}3 \\
27,30,32\end{array}$ & $\begin{array}{c}1 \\
27\end{array}$ & $\begin{array}{c}1 \\
32\end{array}$ & - & $\begin{array}{c}1 \\
30\end{array}$ & 67 & Inconsistent \\
\hline $\begin{array}{l}\text { Upper extremities } \\
\text { Kinematics }\end{array}$ & $\begin{array}{c}1 \\
33\end{array}$ & - & $\begin{array}{c}1 \\
33\end{array}$ & - & - & 100 & Insufficient \\
\hline \multicolumn{8}{|l|}{ Pelvis and hip } \\
\hline Sagittal & $\begin{array}{c}7 \\
24,28,29,34,37,39,41\end{array}$ & $\begin{array}{c}4 \\
28,34,39,41\end{array}$ & - & $\begin{array}{c}3 \\
24,29,37\end{array}$ & - & 57 & Inconsistent \\
\hline Frontal & $\begin{array}{c}8 \\
28,29,34,37,39-41,47\end{array}$ & $\begin{array}{c}4 \\
28,34,40,47\end{array}$ & - & $\begin{array}{c}4 \\
29,37,39,41\end{array}$ & - & 50 & Inconsistent \\
\hline Transversal & $\underset{28,34}{2}$ & $\underset{28,34}{2}$ & - & - & - & 100 & Strong diff. \\
\hline $\begin{array}{l}\text { Kinetics } \\
\text { Sagittal }\end{array}$ & $\begin{array}{c}6 \\
28,29,36-38,69\end{array}$ & $\begin{array}{c}5 \\
28,29,36-38\end{array}$ & $\begin{array}{l}1 \\
69\end{array}$ & - & - & 100 & Strong diff. \\
\hline Frontal & $\begin{array}{c}4 \\
29,36-38\end{array}$ & $\begin{array}{c}4 \\
29,36-38\end{array}$ & - & - & - & 100 & Strong diff \\
\hline Transversal & $\underset{36,38}{2}$ & $\begin{array}{c}1 \\
38\end{array}$ & - & $\begin{array}{c}1 \\
36\end{array}$ & - & 50 & Inconsistent \\
\hline Contact force & $\begin{array}{c}1 \\
39\end{array}$ & $\begin{array}{c}1 \\
39\end{array}$ & - & - & - & 100 & Insufficient \\
\hline \multicolumn{8}{|l|}{ Knee } \\
\hline Sagittal & $\begin{array}{c}9 \\
24,28,29,34,37,41,45,47,70\end{array}$ & $\begin{array}{c}5 \\
28,29,34,41,47\end{array}$ & $\begin{array}{c}1 \\
70\end{array}$ & $\begin{array}{c}3 \\
24,37,45\end{array}$ & - & 66 & Inconsistent \\
\hline Frontal & $\begin{array}{c}4 \\
29,37,40,41\end{array}$ & $\begin{array}{c}3 \\
29,40,41\end{array}$ & - & $\begin{array}{c}1 \\
37\end{array}$ & - & 75 & Strong diff. \\
\hline Transversal & $\begin{array}{c}1 \\
34\end{array}$ & $\begin{array}{c}1 \\
34\end{array}$ & - & - & - & 100 & Insufficient \\
\hline $\begin{array}{l}\text { Kinetics } \\
\text { Sagittal }\end{array}$ & $\begin{array}{c}7 \\
29,32,36-38,42,70\end{array}$ & $\begin{array}{c}5 \\
29,36-38,42\end{array}$ & - & - & $\underset{32,70}{2}$ & 71 & $\begin{array}{l}\text { Moderate } \\
\text { diff. }\end{array}$ \\
\hline Frontal & $\begin{array}{c}7 \\
29,36-38,42,45,70\end{array}$ & $\underset{36-38,42,45}{5}$ & $\begin{array}{c}1 \\
70\end{array}$ & $\begin{array}{c}1 \\
29\end{array}$ & - & 86 & Strong diff. \\
\hline Transversal & $\begin{array}{c}1 \\
37\end{array}$ & $\begin{array}{c}1 \\
37\end{array}$ & - & - & - & 100 & Insufficient \\
\hline
\end{tabular}




\begin{tabular}{|c|c|c|c|c|c|c|c|}
\hline Contact force & $\begin{array}{c}2 \\
44,45\end{array}$ & $\begin{array}{c}1 \\
45\end{array}$ & $\begin{array}{c}1 \\
44\end{array}$ & - & - & 100 & $\begin{array}{c}\text { Moderate } \\
\text { diff. }\end{array}$ \\
\hline \multicolumn{8}{|l|}{ Ankle and foot } \\
\hline Sagittal & $\begin{array}{c}7 \\
24,28,29,34,37,41,56\end{array}$ & $\begin{array}{c}4 \\
24,28,41,56\end{array}$ & - & $\begin{array}{c}3 \\
29,34,37\end{array}$ & - & 57 & Inconsistent \\
\hline Frontal & $\begin{array}{c}6 \\
29,34,37,40,41,56\end{array}$ & $\begin{array}{c}3 \\
34,40,56\end{array}$ & - & $\begin{array}{c}3 \\
29,37,41\end{array}$ & - & 50 & Inconsistent \\
\hline Transversal & $\begin{array}{c}3 \\
34,37,56\end{array}$ & $\underset{34,56}{2}$ & - & $\begin{array}{c}1 \\
37\end{array}$ & - & 67 & Inconsistent \\
\hline \multicolumn{8}{|l|}{ Kinetics } \\
\hline Sagittal & $\begin{array}{c}7 \\
28,29,32,36-38,70\end{array}$ & $\begin{array}{c}4 \\
28,36-38\end{array}$ & $\begin{array}{c}1 \\
70\end{array}$ & $\begin{array}{c}1 \\
29\end{array}$ & $\begin{array}{c}1 \\
69\end{array}$ & 71 & $\begin{array}{c}\text { Moderate } \\
\text { diff. }\end{array}$ \\
\hline Frontal & $\begin{array}{c}2 \\
29,37\end{array}$ & $\begin{array}{c}1 \\
37\end{array}$ & - & $\begin{array}{c}1 \\
29\end{array}$ & - & 50 & Inconsistent \\
\hline Transversal & $\begin{array}{c}1 \\
37\end{array}$ & $\begin{array}{l}1 \\
37\end{array}$ & - & - & - & 100 & Insufficient \\
\hline \multicolumn{8}{|l|}{ Centre of mass } \\
\hline Velocity/acceleration & $\begin{array}{c}1 \\
46\end{array}$ & - & $1^{46}$ & - & - & 100 & Insufficient \\
\hline Lateral displacement & $\begin{array}{l}1 \\
46\end{array}$ & - & $1^{46}$ & - & - & 100 & Insufficient \\
\hline \multicolumn{8}{|l|}{ Muscle Activation } \\
\hline Psoas and iliacus & $\begin{array}{c}1 \\
39\end{array}$ & - & - & $\begin{array}{c}1 \\
39\end{array}$ & - & 100 & Insufficient \\
\hline Gluteus complex & $\begin{array}{c}2 \\
39,47\end{array}$ & $\begin{array}{c}1 \\
47\end{array}$ & - & $\begin{array}{c}1 \\
39\end{array}$ & - & 50 & Inconsistent \\
\hline Quadriceps & $\begin{array}{c}4 \\
39,44,47,49\end{array}$ & - & $\begin{array}{c}1 \\
44\end{array}$ & $\begin{array}{c}3 \\
39,47,49\end{array}$ & - & 75 & $\begin{array}{l}\text { Strong no } \\
\text { diff. }\end{array}$ \\
\hline Hamstring & $\begin{array}{c}3 \\
39,44,49\end{array}$ & - & $\begin{array}{c}1 \\
44\end{array}$ & $\begin{array}{c}2 \\
39,49\end{array}$ & - & 67 & $\begin{array}{l}\text { Moderate } \\
\text { no diff. }\end{array}$ \\
\hline Gastrocnemius/soleus & $\begin{array}{c}2 \\
44,47\end{array}$ & $\begin{array}{c}1 \\
47\end{array}$ & $\begin{array}{c}1 \\
44\end{array}$ & - & - & 100 & $\begin{array}{c}\text { Moderate } \\
\text { diff. }\end{array}$ \\
\hline Tibialis anterior & $\begin{array}{c}1 \\
49\end{array}$ & - & - & $\begin{array}{c}1 \\
49\end{array}$ & - & 100 & Insufficient \\
\hline
\end{tabular}

Note that the percentage of consistency is calculated by dividing the number of studies reporting significant or no significant differences (depending on where the evidence points) by the total number of studies reporting this specific gait biomechanical parameter. Consistent findings ( $\geq 75 \%$ of results showed significant or no significant differences). $N$ studies: number of studies reporting a biomechanical parameter; diff: difference between children and adolescents with overweight/obese vs their normal-weight peers. 


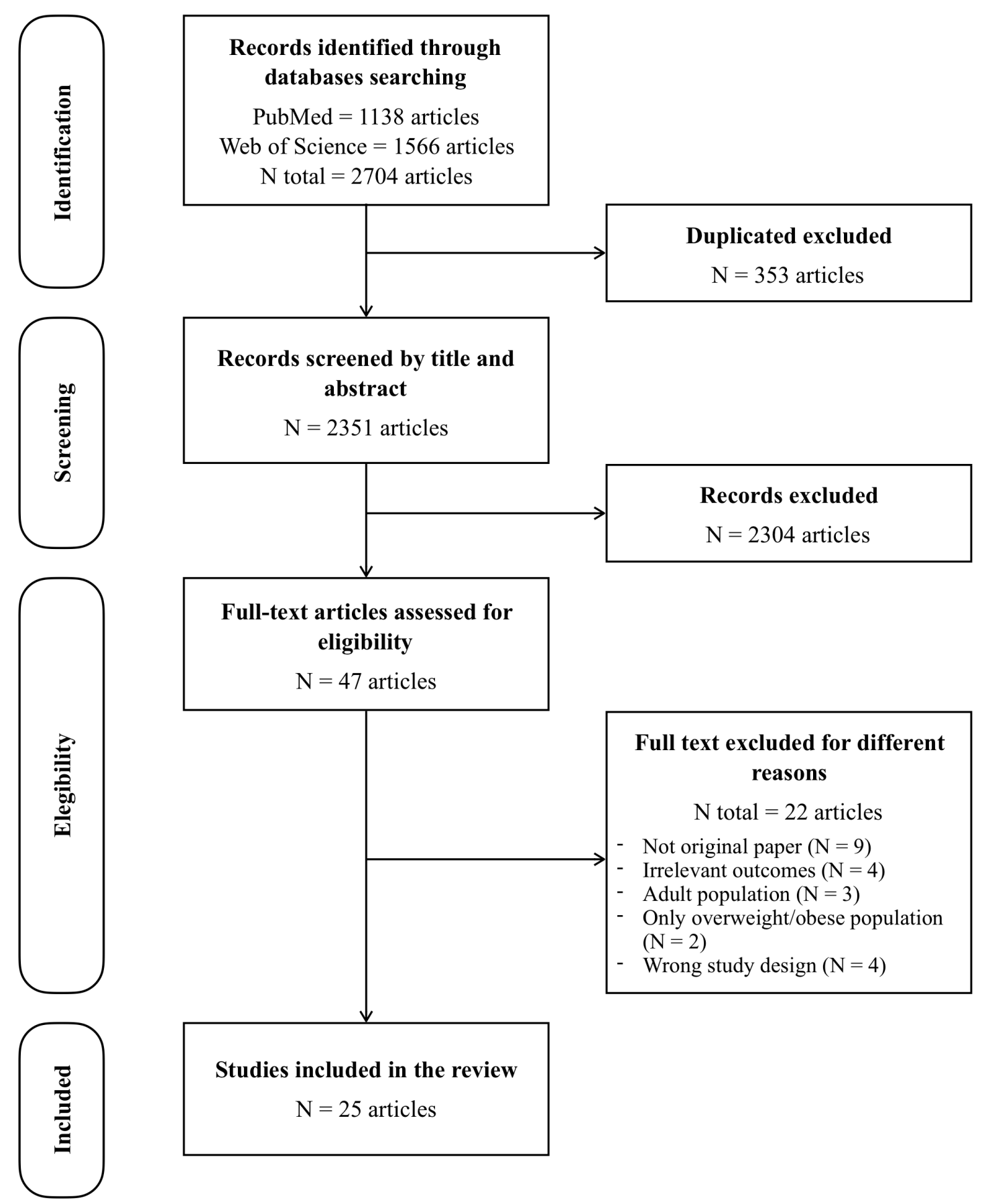

Figure 1. Preferred Reporting Items for Systematic Reviews and Meta-Analyses (PRISMA) flow diagram of study selection, inclusion and exclusion of studies. 


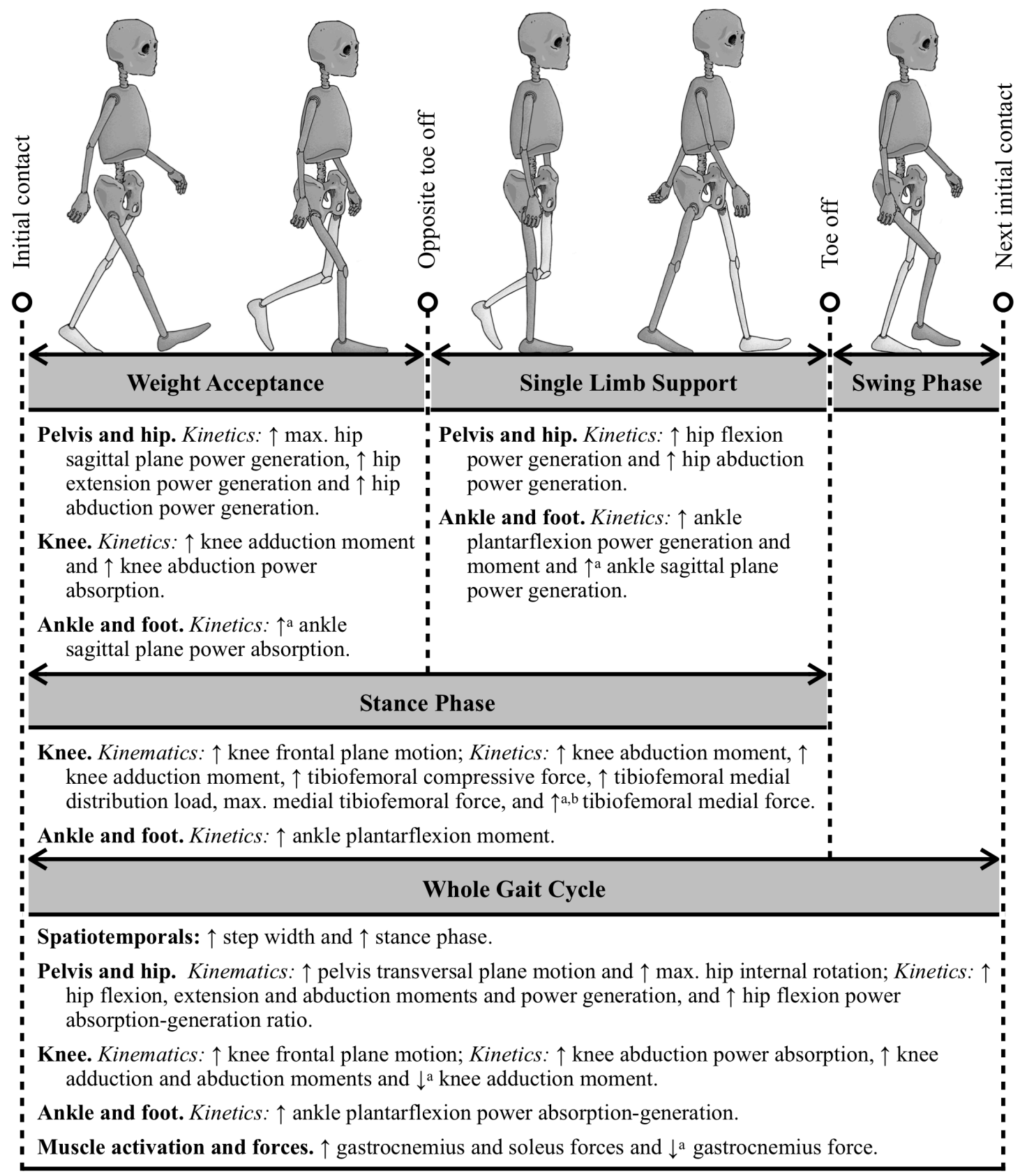

Figure 2. Schematic summary of main gait biomechanical differences between children and adolescents with overweight/obesity and normal-weight divided by key gait phases.

$\uparrow$ : indicates significantly higher values in children and adolescents with overweight/obesity versus their normal-weight peers; $\downarrow$ indicates significantly lower values in children and adolescents with overweight/obesity versus their normal-weight peers; ${ }^{a}$ normalized for body mass $(\mathrm{kg})$; ${ }^{\mathrm{b}}$ normalized for the physis cross-sectional area $\left(\mathrm{cm}^{2}\right)$ 